\title{
A maximização da assimetria na seleção de carteiras de investimento e a generalização do modelo para momentos ímpares de ordem superior
}

\author{
Patricia R. Martins ${ }^{1}$ \\ Ccomp/UERJ, Rio de Janeiro, RJ \\ Patrícia N. da Silva² \\ Ccomp/UERJ, Rio de Janeiro, RJ \\ Carlos F. Vasconcellos ${ }^{3}$ \\ Ccomp/UERJ, Rio de Janeiro, RJ
}

\begin{abstract}
Resumo. Neste trabalho, apresentamos um modelo de maximização da assimetria de um portfólio quando fixados os dois primeiros momentos, considerando um ativo livre de risco e permitindo vendas a descoberto. Deduzimos propriedades geométricas de suas soluções. A partir deste modelo generalizamos os resultados para maximização de momentos ímpares quaisquer, dados os mesmos parâmetros, retorno e variância.
\end{abstract}

Palavras-chave. Seleção de carteiras de investimento, Maximização da assimetria, Momentos de ordem superior

\section{Introdução}

O interesse pela otimização de um portfólio onde são considerados momentos de ordem superior vem se renovando nos últimos anos. Contribuições, como as verificadas nos trabalhos de Athayde e Flores [1] e [2], lançam luz a uma série de questões que antes impediam o desenvolvimento matemático desta importante abordagem para seleção de carteiras de investimento. Eles utilizam uma nova notação para representar qualquer momento tensor relacionado a um vetor aleatório multivariado de retornos de ativos que pode ser usada em um contexto de maximização de utilidade ou se portfólios ótimos são definidos por relações de preferência.

Desde Martins [4], desenvolvemos um trabalho de pesquisa com modelos de seleção de carteira de investimento sob a ótica da maximização da utilidade, explorando a dualidade verificada na natureza deste problema e a necessidade de considerar momentos de ordem superior. Segundo Athayde e Flores em [1], a notação por eles introduzida trata o problema em um cenário absolutamente geral, o que significa tanto na ordem máxima $p$ dos momentos de interesse do portfólio, quanto na representação sistemática da assimetria ou outros tensores de ordem superior, sendo esta última crucial! E acreditam que suas implicações ainda não foram totalmente exploradas. Embora o conjunto de todos co-momentos possa se tornar rapidamente proibitivo - o que, além de outras questões, pode representar dificuldades de estimativa econométrica para a aplicação -, e hipóteses simplificadoras sobre seu padrão geralmente serão impostas na prática, é importante analisar a

\footnotetext{
${ }^{1}$ patriciarm75@yahoo.com.br

2 nunes@ime.uerj.br

3 cfredvasc@gmail.com
} 
solução geral para o problema, independentemente de outras suposições que possam ser impostas. O rigor para a ordenação preferencial de portfólios dada por Scott e Horvath (1980), pode levar a resultados mais interessantes no contexto estrito de otimização de portfólio. Em [1], podemos encontrar a minimização generalizada para qualquer momento par de um dado portfólio com retorno e assimetria fixados, bem como a discussão de propriedades geométricas destes modelos.

Lidar com momentos de ordem superior pode representar um desafio algébrico. Dado um vetor aleatório $n$-dimensional, o conjunto de seus momentos de ordem $p$ pode ser representado por um tensor. Athayde e Flores [1] desenvolveram uma notação especial que permite que todas as operações necessárias entre os momentos sejam realizadas através de cálculo matricial. Em sua notação um tensor de $p$-ésimos momentos, com $n^{p}$ elementos é transformado em uma matriz de ordem $n \times n^{p-1}$. No caso da assimetria, por exemplo, o tensor de terceiros momentos é transformado em uma matriz $n \times n^{2}$, separando o cubo em camadas $n \times n$ que serão dispostas lado a lado,

$$
M_{3}=\left|\begin{array}{ccccccc}
\sigma_{111} & \cdots & \sigma_{11 n} & \cdots & \sigma_{n 11} & \cdots & \sigma_{n 1 n} \\
\vdots & \ddots & \vdots & \vdots & \vdots & \ddots & \vdots \\
\sigma_{1 n 1} & \cdots & \sigma_{1 n n} & \cdots & \sigma_{n n 1} & \cdots & \sigma_{n n n}
\end{array}\right|,
$$

de modo que a assimetria do retorno da carteira pode então ser representada por:

$$
\alpha^{t} M_{3}(\alpha \otimes \alpha)
$$

em que $\otimes$ denota o produto de Kronecker, $M_{3}$ representa a matriz que contém as co-assimetrias do vetor aleatório de $n$ ativos e $\alpha \in \mathbb{R}^{n}$ seus pesos correspondentes.

Neste trabalho, apresentamos um modelo para maximizar um momento ímpar qualquer sujeito a restrição dos dois primeiros momentos, tendo em vista que os investidores gostam de momentos ímpares e não gostam de pares. E discutimos a propriedade homotética presente na estrutura que surge a partir desta otimização no espaço dos momentos. Ao longo de todo o texto, assumimos que os gradientes das restrições nos pontos ótimos são linearmente independentes.

Nas Seções 2 e 3, apresentamos a otimização da variância considerando o primeiro e o terceiro momentos desejados do portfólio, uma propriedade homotética e a generalização destes resultados para momentos de ordem par quaisquer, observadas em [1]. Na Seção 4, desenvolvemos o modelo de otimização da assimetria considerando os dois primeiros momentos e mostramos que a propriedade homotética também se verifica. A Seção 5 generaliza os resultados para momentos de ordem ímpar quaisquer. Em nossa conclusão, explicamos como nossos resultados complementam [1], dando maior relevância a abordagem dos modelos duais.

\section{Variância mínima sujeita aos dois primeiros momentos ímpares}

O material desta seção se baseia em Athayde e Flores [1], onde é encontrada uma análise profunda do problema de portfólio ótimo considerados os três primeiros momentos.

Minimizar a variância, para um determinado retorno médio e assimetria, equivale a encontrar a solução para o problema:

$$
\min _{\alpha} L=\alpha^{t} M_{2} \alpha+\lambda_{1}\left(\sigma_{p^{3}}-\alpha^{t} M_{3}(\alpha \otimes \alpha)\right)+\lambda_{2}\left[E\left(r_{p}\right)-\left(\alpha^{t} M_{1}+\left(1-\alpha^{t}[1]\right) r_{f}\right)\right],
$$

em que $M_{1}, M_{2}$ e $M_{3}$ são respectivamente as matrizes relacionadas aos tensores de primeiro, segundo e terceiro momentos verificados para os $n$ ativos de risco, $\alpha$ é o vetor dos $n$ pesos do portfólio - onde são permitidas vendas a descoberto -, $r_{f}$ a taxa de retorno sem risco, [1] é um vetor $n \times 1$ 
de 1's e os lambdas são multiplicadores de Lagrange.

Denotando por $R=E\left[r_{p}\right]-r_{f}$ e $x=M_{1}-[1] r_{f}$, temos que $R$ será o retorno excedente da carteira representado pela equação $\alpha^{t} x=R$ onde a soma dos pesos é igual a 1 . Assim as condições de primeira ordem são:

$$
\left\{\begin{array}{l}
2 M_{2} \alpha=3 \lambda_{1} M_{3}(\alpha \otimes \alpha)+\lambda_{2} x \\
\alpha^{t} M_{3}(\alpha \otimes \alpha)=\sigma_{p^{3}} \\
\alpha^{t} x=R
\end{array} .\right.
$$

A solução para (1) é encontrada resolvendo o sistema de $n$-equações não lineares,

$$
M_{2} \alpha=\frac{A_{0} \sigma_{p^{3}}-A_{2} R}{A_{0} A_{4}-\left(A_{2}\right)^{2}} M_{3}(\alpha \otimes \alpha)+\frac{A_{4} R-A_{2} \sigma_{p^{3}}}{A_{0} A_{4}-\left(A_{2}\right)^{2}} x
$$

em que os escalares:

$$
\begin{aligned}
& A_{0}=x^{t} M_{2}^{-1} x \\
& A_{2}=x^{t} M_{2}^{-1} M_{3}(\alpha \otimes \alpha) \\
& A_{4}=(\alpha \otimes \alpha)^{t} M_{3}^{t} M_{2}^{-1} M_{3}(\alpha \otimes \alpha) ;
\end{aligned}
$$

tem subscritos correspondentes ao seu grau de homogeneidade como funções reais do vetor $\alpha$. Em particular, $A_{0}$ e $A_{4}$ são positivos, uma vez que a inversa da matriz de covariância é positiva definida.

Multiplicando a Equação (2) pelas próprias soluções $\alpha^{t}$, obtêm-se a variância ótima:

$$
\sigma_{p^{2}}=\frac{A_{4} R^{2}-2 A_{2} R \sigma_{p^{3}}+A_{0}\left(\sigma_{p^{3}}\right)^{2}}{A_{0} A_{4}-\left(A_{2}\right)^{2}} .
$$

A proposição a seguir foi obtida por Athayde e Flôres [2].

Proposição 2.1. Para um dado $k$ positivo, seja $\bar{\alpha}$ o portfólio de variância mínima quando $R=1$ e $\sigma_{p^{3}}=k^{3}$, e $\bar{\sigma}_{p^{2}}$ a variância mínima correspondente, então, para todo portfólio ótimo relacionado ao par assimetria/retorno tal que $\sigma_{p^{3}}=k^{3} R^{3}$, uma solução para (1) será $\alpha=\bar{\alpha} R$, com variância minima correspondente $\sigma_{p^{2}}=\bar{\sigma}_{p^{2}} R^{2}$.

\section{A generalização para momentos pares de ordem superior}

Dado um vetor de pesos $\alpha \in \mathbb{R}^{n}$, na notação introduzida por Athayde e Flores [1], o $p$-ésimo momento do portfólio com estes pesos é representado por

$$
\alpha^{t} M_{p}(\alpha \otimes \alpha \otimes \alpha \cdots \otimes \alpha) \equiv \alpha^{t} M_{p} \alpha^{\otimes(p-1)},
$$

em que $\otimes$ denota o produto de Kronecker e $M_{p}$ representa a matriz que contém os $p$-ésimos momentos do vetor aleatório de $n$ ativos.

Para o caso geral de minimizar um momento par de ordem $p$ quando fixados os dois primeiros momentos ímpares, o problema será:

$$
\min _{\alpha} L=\alpha^{t} M_{p} \alpha^{\otimes(p-1)}+\lambda\left(\sigma_{p^{3}}-\alpha^{t} M_{3} \alpha^{\otimes 2}\right)+\gamma\left(R-\alpha^{t} x\right) .
$$


Logo as condições de primeira ordem serão:

$$
\left\{\begin{array}{l}
p M_{p} \alpha^{\otimes(p-1)}=3 \lambda M_{3} \alpha^{\otimes 2}+\gamma x \\
\alpha^{t} M_{3} \alpha^{\otimes 2}=\sigma_{p^{3}} \\
\alpha^{t} x=R
\end{array}\right.
$$

Notando que $M_{p} \alpha^{\otimes(p-1)}=M_{p}\left(\alpha^{\otimes(p-2)} \otimes I_{n}\right) \alpha$, e que a matriz $M_{p}\left(\alpha^{\otimes(p-2)} \otimes I_{n}\right)$ é simétrica e definida positiva, o seguinte sistema pode ser formado a partir de (5a) para obter os valores dos multiplicadores:

$$
\left\{\begin{array}{l}
p R=3 \lambda x^{t}\left(M_{p} \alpha^{\otimes(p-2)} \otimes I_{n}\right)^{-1} M_{3} \alpha^{\otimes 2}+\gamma x^{t}\left(M_{p} \alpha^{\otimes(p-2)} \otimes I_{n}\right)^{-1} x \\
p \sigma_{p^{3}}=3 \lambda\left(M_{3} \alpha^{\otimes 2}\right)^{t}\left(M_{p} \alpha^{\otimes(p-2)} \otimes I_{n}\right)^{-1} M_{3} \alpha^{\otimes 2}+\gamma\left(M_{3} \alpha^{\otimes 2}\right)^{t}\left(M_{p} \alpha^{\otimes(p-2)} \otimes I_{n}\right)^{-1} x .
\end{array}\right.
$$

Definindo

$$
\begin{aligned}
& B_{2-p}=x^{t}\left[M_{p}\left(\alpha^{\otimes} I_{n}\right)^{-1}\right] x, \\
& B_{4-p}=x^{t}\left[M_{p}\left(\alpha^{\otimes} I_{n}\right)^{-1}\right] M_{3}(\alpha \otimes \alpha) \quad \mathrm{e} \\
& B_{6-p}=\left(\alpha^{\otimes 2}\right)^{t} M_{3}^{t}\left[M_{p}\left(\alpha^{\otimes} I_{n}\right)\right]^{-1} M_{3} \alpha^{\otimes 2},
\end{aligned}
$$

com os subscritos correspondentes ao grau generalizado de homogeneidade com respeito ao vetor de pesos, a solução final vem do sistema:

$$
\left(B_{2-p} B_{6-p}-\left(B_{4-p}\right)^{2}\right) M_{p} \alpha^{\otimes(p-1)}=\left(B_{2-p} \sigma_{p^{3}}-B_{4-p} R\right) M_{3} \alpha^{\otimes 2}+\left(B_{6-p} R-B_{4-p} \sigma_{p^{3}}\right) x .
$$

O p-ésimo momento do portfólio ótimo será dado por:

$$
\sigma_{p^{p}}=\frac{B_{6-p} R^{2}-2 B_{4-p} R \sigma_{p^{3}}+B_{2-p}\left(\sigma_{p^{3}}\right)^{2}}{B_{2-p} B_{6-p}-\left(B_{4-p}\right)^{2}} .
$$

A proposição a seguir foi obtida por Athayde e Flôres [1].

Proposição 3.1. Para um dado $k$ positivo, seja $\bar{\alpha}$ o portfólio que minimiza o momento par de ordem $p$ quando $R=1$ e $\sigma_{p^{3}}=k^{3}$, e $\bar{\sigma}_{p^{p}}$ o p-ésimo momento mínimo correspondente, então, para todo portfólio ótimo relacionado ao par assimetria/retorno tal que $\sigma_{p^{3}}=k^{3} R^{3}$, uma solução para (4) será $\alpha=\bar{\alpha} R$, com p-ésimo momento mínimo correspondente $\sigma_{p^{p}}=\bar{\sigma}_{p^{p}} R^{p}$.

\section{Carteiras de Assimetria Máxima}

Para obtermos os mesmos resultados para momentos de ordem ímpar, inicialmente vamos maximizar a assimetria fixando os dois primeiros momentos, retorno e variância:

$$
\begin{gathered}
\max _{\alpha} L=\alpha^{t} M_{3}(\alpha \otimes \alpha)+\gamma_{1}\left(\sigma_{p^{2}}-\alpha^{t} M_{2} \alpha\right)+\gamma_{2}\left(R-\alpha^{t} x\right), \\
\left\{\begin{array}{l}
3 M_{3}(\alpha \otimes \alpha)=2 \gamma_{1} M_{2} \alpha+\gamma_{2} x \\
\alpha^{t} M_{2} \alpha=\sigma_{p^{2}} \\
\alpha^{t} x=R
\end{array}\right.
\end{gathered}
$$


Determinamos os multiplicadores obtendo as equações:

$$
\left\{\begin{array}{l}
2 \gamma_{1} \sigma_{p^{2}}+\gamma_{2} R=3 \alpha^{t} M_{3}(\alpha \otimes \alpha) \\
2 \gamma_{1} R+\gamma_{2} A_{0}=3 A_{2}
\end{array}\right.
$$

em que

$$
\begin{aligned}
& A_{0}=x^{t} M_{2}^{-1} x, \\
& A_{2}=x^{t} M_{2}^{-1} M_{3}(\alpha \otimes \alpha),
\end{aligned}
$$

e encontramos o seguinte sistema de $n$ equações não lineares em $\alpha$ que satisfaz a equação de Lagrange:

$$
\left(\frac{A_{0} \alpha^{t} M_{3}(\alpha \otimes \alpha)-A_{2} R}{\sigma_{p^{2}} A_{0}-R^{2}}\right) \alpha=M_{2}^{-1} M_{3}(\alpha \otimes \alpha)-\left(\frac{\sigma_{p^{2}} A_{2}-\alpha^{t} M_{3}(\alpha \otimes \alpha) R}{\sigma_{p^{2}} A_{0}-R^{2}}\right) M_{2}^{-1} x .
$$

A partir da solução dada pela Equação (7), obtemos a assimetria ótima para a maximização do terceiro momento. Esta assimetria associada a carteira eficiente irá indicar se os parâmetros fixados possibilitam a dualidade com o problema de minimizar a variância.

Neste trabalho, obtivemos o seguinte resultado de propriedade de homotetia, semelhante àquele verificado para o conjunto de variância mínima, para a maximização da assimetria.

Proposição 4.1. Para um dado $k$ positivo e maior ou igual a $\frac{1}{\sqrt{A_{0}}}$, seja $\bar{\alpha}$ o portfólio de assimetria máxima (mínima) quando $R=1$ e $\sigma_{p^{2}}=k^{2}$, e $\bar{\sigma}_{p^{3}}$ a assimetria máxima (minima) correspondente, então, para todo portfólio ótimo relacionado ao par variância/retorno tal que $\sigma_{p^{2}}=k^{2} R^{2}$, uma solução para (6) será $\alpha=\bar{\alpha} R$, com assimetria máxima correspondente $\sigma_{p^{3}}=\bar{\sigma}_{p^{3}} R^{3}$.

A seguir generalizamos os resultados obtidos para qualquer momento de ordem ímpar dados o retorno esperado e a variância.

\section{Generalizando os momentos ímpares de ordem superior}

Dado um vetor de pesos $\alpha \in \mathbb{R}^{n}$, na notação introduzida por Athayde e Flores [1], o $q$-ésimo momento do portfólio com estes pesos é representado por

$$
\alpha^{t} M_{q}(\alpha \otimes \alpha \otimes \alpha \cdots \otimes \alpha) \equiv \alpha^{t} M_{q} \alpha^{\otimes(q-1)},
$$

em que $\otimes$ denota o produto de Kronecker e $M_{q}$ representa a matriz que contém os $q$-ésimos momentos do vetor aleatório de $n$ ativos.

Para o caso geral de maximizar um momento ímpar de ordem $q$ quando fixados os dois primeiros momentos, o problema será:

$$
\begin{gathered}
\max _{\alpha} L=\alpha^{t} M_{q} \alpha^{\otimes(q-1)}+\lambda\left(\sigma_{p^{2}}-\alpha^{t} M_{2} \alpha\right)+\gamma\left(R-\alpha^{t} x\right), \\
\left\{\begin{array}{l}
q M_{q} \alpha^{\otimes(q-1)}=2 \lambda M_{2} \alpha+\gamma x \\
\alpha^{t} M_{2} \alpha=\sigma_{p^{2}} \\
\alpha^{t} x=R
\end{array}\right.
\end{gathered}
$$


Como estamos lidando com momentos de ordem ímpar, a matriz $M_{2}$ não se altera. De modo que definindo

$$
\begin{aligned}
& A_{0}=x^{t} M_{2}^{-1} x, \\
& A_{q}=x^{t} M_{2}^{-1} M_{q} \alpha^{\otimes(q-1)},
\end{aligned}
$$

obtemos as seguintes equações:

$$
\left\{\begin{array}{l}
2 \lambda \sigma_{p^{2}}+\gamma R=q \alpha^{t} M_{q} \alpha^{\otimes(q-1)} \\
2 \lambda R+\gamma A_{0}=q A_{q}
\end{array} .\right.
$$

Obtemos ainda o sistema de $n$ equações não lineares em $\alpha$, que satisfaz a equação de Lagrange, e a partir dele calculamos o $q$-ésimo momento máximo.

Neste trabalho, obtivemos também o seguinte resultado de propriedade de homotetia, semelhante ao obtido em [1].

Proposição 5.1. Para um dado $k$ positivo, seja $\bar{\alpha}$ o portfólio que maximiza o momento ímpar de ordem q quando $R=1$ e $\sigma_{p^{2}}=k^{2}$, e $\bar{\sigma}_{q^{q}}$ o q-ésimo momento máximo correspondente, então, para todo portfólio ótimo relacionado ao par variância/retorno tal que $\sigma_{p^{2}}=k^{2} R^{2}$, uma solução para (8) será $\alpha=\bar{\alpha} R$, com q-ésimo momento máximo correspondente $\sigma_{q^{q}}=\bar{\sigma}_{q^{q}} R^{q}$.

\section{Conclusões}

A generalização para momentos ímpares de um dado portfólio, complementa o método geral proposto em [1] para tratar a escolha do portfólio em um contexto de momentos de ordem superior, vista como uma vantagem inquestionável. Uma destas vantagens está na caracterização final do conjunto eficiente de portfólios a partir da dualidade, auxiliando na identificação dos pontos eficientes. A garantia de existência e mais informações sobre o conjunto de soluções são nosso objeto atual de estudo com resultados conclusivos e outros em estágio avançado. Além disso, o teste final dos ganhos obtidos com momentos de ordem superior depende de extensas aplicações práticas dos novos resultados. Estes, por sua vez, exigem ferramentas de software adequadas para resolver os sistemas não lineares e os problemas de otimização envolvidos. Um melhor conhecimento das superfícies relacionadas a elas pode melhorar muito o design do software.

\section{$7 \quad$ Agradecimentos}

Agradecemos a FAPERJ pelo apoio financeiro.

\section{Referências}

[1] Athayde, G.M. e Flores R.G.Jr. On Certain Geometric Aspects of Portfolio Optimisation with Higher Moments, Ensaios Econômicos, 453:1-23, 2002. ISSN: 0104-8910.

[2] Athayde, G.M. e Flores R.G.Jr. Finding a maximum skewness portfolio - a general solution to three-moments portfolio choice, Journal of Economic Dynamics and Control, 28:1335-1352, 2004. DOI: 10.1016/S0165-1889(02)00084-2. 
[3] Markowitz, H. Portfolio Selection, The Journal of Finance 7:77-91, 1952. DOI: 10.1111/j.15406261.1952.tb01525.x.

[4] Martins, P. R. Aplicação de Teorema de Ponto Fixo a um Modelo de Seleção de Carteiras de Investimento, Dissertação de Mestrado, UERJ, 2015.

[5] Scott, R. C. e Horvath, P. A. On the Direction of Preference for Moments of Higher Order than the Variance. The Journal of Finance, volume 35, n. 4:915-919, 1980. DOI: 10.1111/j.15406261.1980.tb03509.x. 